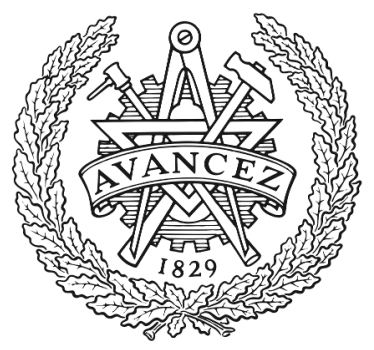

CHALMERS

UNIVERSITY OF TECHNOLOGY

\title{
Energy-Efficient High-Throughput Staircase Decoders
}

Downloaded from: https://research.chalmers.se, 2023-04-26 14:37 UTC

Citation for the original published paper (version of record):

Fougstedt, C., Larsson-Edefors, P. (2018). Energy-Efficient High-Throughput Staircase Decoders. 2018 Optical Fiber Communications Conference and Exposition, OFC 2018 - Proceedings: 1-3. http://dx.doi.org/10.1364/OFC.2018.TU3C.6

N.B. When citing this work, cite the original published paper. 


\title{
Energy-Efficient High-Throughput Staircase Decoders
}

\author{
Christoffer Fougstedt and Per Larsson-Edefors \\ Dept. of Computer Science and Engineering, \\ Chalmers University of Technology, SE-412 96 Göteborg, Sweden \\ chrfou@chalmers.se
}

\begin{abstract}
:
We introduce staircase decoder implementations achieving up to 1-Tb/s throughput with energy dissipation of $1.2 \mathrm{pJ} /$ information bit. The implementations are estimated to achieve $>10.5 \mathrm{~dB}$ of net coding gain depending on the configuration.

OCIS codes: (060.0060) Fiber optics and optical communication; (060.2330) Fiber optics communications
\end{abstract}

\section{Introduction}

Staircase codes [1] have attracted considerable interest in the research community. While staircase codes have been considered at an algorithmic level [2,3], to the best of our knowledge, no studies on application-specific integrated circuit (ASIC) implementation aspects have been published in the open literature. In this paper, we will describe and evaluate circuit implementations of a staircase decoder. Using a window to store staircase data blocks and a set of Bose-Chaudhuri-Hocquenghem (BCH) decoders for the component codes, the staircase decoder design we propose can support a wide range of implementations in response to different throughput needs.

The actual probability of performing correction of an error in a component code-word depends on the position of the code-word in the window and the number of iterations performed. Since errors are successively corrected, the component decoders are more active in the front-end of the window and during the first iterations. As power dissipation depends on signal switching statistics, it is crucial to recognize the significant spatial and temporal variation in switching activities when developing an energy-efficient decoder implementation. Here, the decoder design benefits greatly from gating of the clock when circuits are idle, to reduce clock power and redundant logic signal switching.

Beside energy efficiency and coding gain, also throughput and latency are critical system parameters. The BCH decoders that we employ in the staircase are an extension of our previous work [4]. They operate in a non-iterative manner which simplifies the design of state machines for staircase control. Additionally, since the component codes can be decoded with low-latency circuits, we are able to achieve very high staircase decoder throughput.

We will first introduce the staircase decoder architecture and the constituent parts, i.e., staircase window and $\mathrm{BCH}$ decoders, with syndrome calculation, key-equation solver and Chien search. Then we will evaluate two implementations with different error correction capabilities and discuss the results that we obtain after synthesizing the implementations to a $28-\mathrm{nm}$ process technology. Finally, we conclude the paper.

\section{ASIC Implementation}

Using the notation $\mathrm{BCH}(n, k, t)$, where $n$ is the block length, $k$ is the number of useful information bits, and $t$ is the number of bit errors that the code can correct, we here use $\mathrm{BCH}(511,484,3)$ and $\mathrm{BCH}(511,475,4)$ codes shortened to 324 and 432 bits respectively, resulting in staircase codes with $20 \%$ overhead, with staircase code block lengths of 26,244 and 46,656, respectively.

\subsection{Component Decoder Implementation}

The implemented staircase decoders are based on the shortened $\mathrm{BCH}$ component codes above. The component codes are decoded using a fully-parallel non-iterative direct-solution algorithm that has been modified to remove Galois field (GF) inversions. While our previous paper [4] introduced such decoders for $t=1$ and $t=2$, here we use recently developed $\mathrm{BCH}$ decoders with $t=3$ and $t=4$. The $\mathrm{BCH}$ decoders are pipelined between the syndrome computation stage, the key-equation solver (KES), and the Chien search; the decoder thus decodes one component code-word in three clock cycles. Since power dissipation depends on signal switching activities, the pipelining registers are clock gated in sequence if a zero-syndrome is detected.

The number of found roots in the Chien search stage is compared to the expected number of roots from the errorlocator polynomial order. If they are not equal, the found roots are discarded. This reduces the miscorrection probability, since a miscorrection can infer errors in the part removed when shortening the code, giving a discrepancy between found roots and polynomial order. 


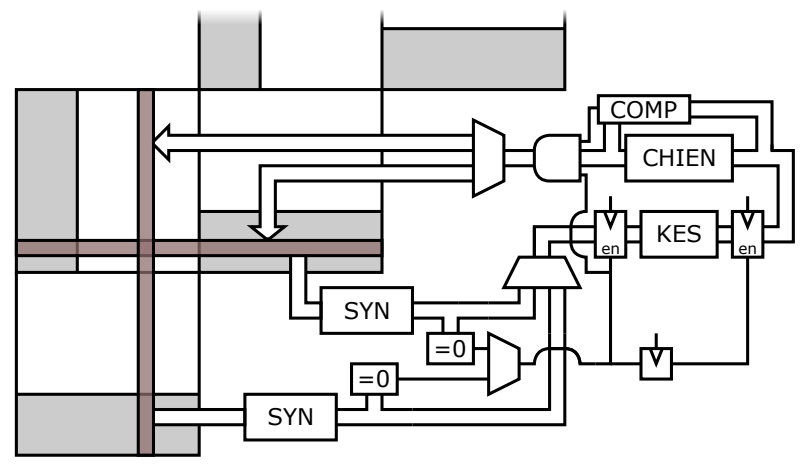

(a) Block diagram of the staircase decoder with single row and column decoder.
Power dissipation distribution

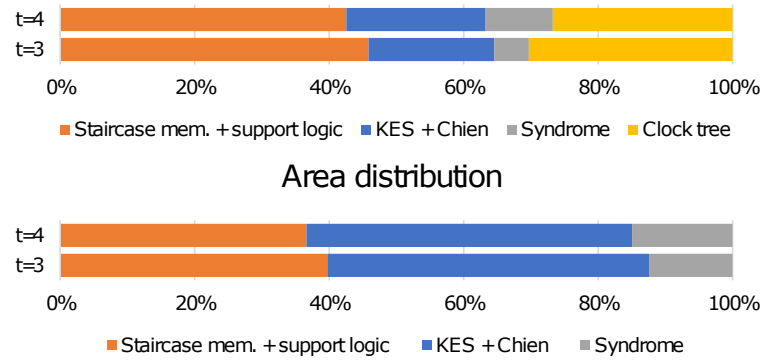

(b) Power dissipation and area distribution for 550-MHz staircase decoder using 5 iterations.

Fig. 1: Decoder circuit architecture with a per-unit area and power dissipation breakdown.

\subsection{Staircase Decoder Implementation}

Power dissipation depends on the number of bits of a word that are switching, rather than the word length itself. One corrected error can cause at most $\left\lceil\log _{2}(n)\right\rceil$ toggles in the syndrome computation tree, so the majority of gates in the XOR-tree remain static. Thus, our implementations use one syndrome computation block per row and per column, resulting in mostly idle logic gates during iterations, with the added benefit of reduced word length in the row-column muxes. The Peterson-based KES and Chien search are shared between one row and one column. Fig. 1a shows a block diagram of one row/column pair.

The implemented staircase decoders operate on a window of 5 blocks. The staircase memory is clock gated when not written to, and each block is clock gated if the row and column syndromes indicate no errors. The window is shifted after a specific number of iterations, which is a system parameter that we can change, and syndromes are then recomputed. While syndrome-recomputation may seem wasteful, it turns out that the syndrome computation dissipates less than $10 \%$ of the total power in the implemented staircase decoders.

Since there are no data dependencies between rows and no dependencies between columns, the implemented staircase decoders iterate between decoding, first, all rows and, then, all columns, instead of decoding the blocks in sequence. This design solution enables an increased throughput and was not found to incur any significant errorcorrection performance degradation in our 5-iteration MATLAB reference implementations.

\subsection{Implementation Area and Power Dissipation}

Fig. 1b shows the power dissipation and area for different circuit blocks of the staircase decoder, using the evaluation methodology outlined in Sec. 3. It is clear from the figure that the power dissipation of component decoders, for reasons outlined in Sec. 1, is not very significant. Also, the figure indicates that power dissipation and area are not strongly correlated, illustrating that algorithm complexity is not a very useful metric for power dissipation in circuits whose signals have very different and varying switching activities. The disconnect between abstract complexity metrics and power dissipation is exacerbated by the addition of a clock tree, which is required for synchronization but which has no direct correspondence in an algorithmic model.

\section{Results and discussion}

The decoders were synthesized using a 28-nm FD-SOI standard-cell flow using regular threshold voltages, slow process corner, $0.9 \mathrm{~V}$, and $125^{\circ} \mathrm{C}$ in Cadence Genus using physical wire models, at a clock rate of $550 \mathrm{MHz}$. Using Cadence Incisive, the resulting netlists were simulated in a VHDL testbench generating uniformly-distributed staircaseencoded input data, which were transmitted over a binary-symmetric channel (BSC), with a bit-error rate (BER) of $10^{-2}$. Beside providing verification of functionality, this simulation generated internal switching activity statistics which were backannotated to the netlist in Cadence Genus to estimate power dissipation using the typical process corner at $25^{\circ} \mathrm{C}$. Clock-tree estimation was performed in Cadence Genus. Synopsys PrimeTime was used to estimate the distribution of data and clock internal power in registers for a $t=3$ decoder implementation using 5 iterations; approximately $70 \%$ of the internal register power was found to be caused by clocking. Static power dissipation accounts for less than $1 \%$ of total power. 
Table 1: Evaluation Results

\begin{tabular}{l|cccc|cccc}
\hline & \multicolumn{4}{|c|}{$t=3$} & \multicolumn{4}{c}{$t=4$} \\
\hline Iterations & 3 & 4 & 5 & 6 & 3 & 4 & 5 & 6 \\
\hline Cell area $\left(\mathbf{m m}^{2}\right)$ & \multicolumn{4}{|c}{7.37} \\
Throughput (Gb/s) & 601 & 463 & 376 & 317 & 1069 & 823 & 668 & 563 \\
Power dissipation (W) & 0.601 & 0.534 & 0.491 & 0.463 & 1.298 & 1.132 & 1.028 & 0.955 \\
Energy per information bit (pJ/bit) & 1.00 & 1.15 & 1.31 & 1.46 & 1.21 & 1.38 & 1.54 & 1.70 \\
Estimated net coding gain (dB) & 10.0 & 10.1 & 10.3 & 10.3 & 10.4 & 10.5 & $>10.5$ & $>10.5$ \\
Block decoding latency (ns) & 181.8 & 236.3 & 290.9 & 345.4 & 181.8 & 236.3 & 290.9 & 345.4 \\
\hline
\end{tabular}

BER simulations were performed on the VHDL staircase decoder implementation using a VHDL BSC testbench, in which the input BER was swept, providing both a functional verification and performance metrics. The resulting output BER was used to estimate coding gain by extrapolation down to $10^{-15}$ using the berfit function in MATLAB. We want to stress that these estimations should be considered as approximations, since excessive runtime limits accurate low-BER statistics. However, the results are consistent with $[2,3]$, taking into account algorithmic differences.

Table 1 presents the implementation data obtained for the two different decoders $(t=3$ and $t=4)$. The number of iterations has an impact on throughput, power dissipation, energy per bit, net coding gain and latency, so we list data for 3-6 iterations.

Focusing on throughput, we can notice that the decoder with $t=4$ can provide very high throughput (in excess of 1 $\mathrm{Tb} / \mathrm{s}$ ) at an energy efficiency of $1.21 \mathrm{pJ} / \mathrm{bit}$. However, the area increase from $t=3$ to $t=4$ is substantial and indicates $t=5$ may not be cost effective from an area utilization perspective. Thanks to extensive clock gating of idle decoder portions, the power dissipation of the $1-\mathrm{Tb} / \mathrm{s}$ implementation is limited to under $1.3 \mathrm{~W}$.

As far as energy efficiency, we can reach as low as $1.0 \mathrm{pJ} / \mathrm{bit}$ for a $600-\mathrm{Gb} / \mathrm{s}$ implementation with a net coding gain of $10.0 \mathrm{~dB}$. As the number of iterations is increased, the power dissipation is decreasing because the signal switching activity is going down for the later iterations. Even though the power dissipation is decreasing with iteration count, the energy efficiency is degrading. This is caused by a reduction in throughput due to an increasing number of iterations. In comparison to recent low-power soft-decision LDPC implementations [5], our staircase decoders achieve better energy-efficiency and higher throughput at the expense of slightly lower coding gain.

\section{Conclusion}

We presented energy-efficient staircase decoder ASIC implementations, which were evaluated in a 28-nm FD-SOI process technology. Depending on decoder configuration, the implementations can achieve up to 1-Tb/s throughput at a power dissipation of $1.3 \mathrm{~W}$, resulting in an energy per information bit of $1.21 \mathrm{pJ} / \mathrm{bit}$. The implemented decoders are estimated to achieve between $10 \mathrm{~dB}$ and $>10.5 \mathrm{~dB}$ net coding gain, and energy per information bit ranges from 1.0 to $1.7 \mathrm{pJ} / \mathrm{bit}$, depending on configuration.

Acknowledgement: The authors would like to thank Dr. Lars Svensson and Dr. Kevin Cushon for fruitful discussions. This work was financially supported by the Knut and Alice Wallenberg Foundation.

\section{References}

[1] B. P. Smith, A. Farhood, A. Hunt, F. R. Kschischang, and J. Lodge, "Staircase codes: FEC for 100 Gb/s OTN," IEEE J. Lightw. Technol. 30, $110-117$ (2012).

[2] C. Häger, A. Graell i Amat, H. D. Pfister, A. Alvarado, F. Brännström, and E. Agrell, "On parameter optimization for staircase codes," in "Opt. Fiber Commun. Conf. (OFC)," (2015).

[3] L. M. Zhang and F. R. Kschischang, "Staircase codes with 6\% to 33\% overhead," IEEE J. Lightw. Technol. 32, 1999-2002 (2014).

[4] C. Fougstedt, K. Szczerba, and P. Larsson-Edefors, "Low-power low-latency BCH decoders for energy-efficient optical interconnects," IEEE J. Lightw. Technol. (to be published).

[5] K. Cushon, P. Larsson-Edefors, and P. Andrekson, "Low-power 400-Gbps soft-decision LDPC FEC for optical transport networks," IEEE J. Lightw. Technol. 34, 4304-4311 (2016). 